\title{
ACHADOS OTONEUROLÓGICOS EM UM CASO DE MIOPATIA INFLAMATÓRIA
}

\section{Otoneurological findings in an inflammatory myopathy case}

\author{
Bianca Simone Zeigelboim (1), Karlin Fabianne Klagenberg ${ }^{(2)}$, Diego Augusto de Brito Malucelli (3), \\ Paulo Breno Noronha Liberalesso ${ }^{(4)}$, Fabiane Paulin ${ }^{(5)}$
}

\begin{abstract}
RESUMO
Tema: a polimiosite é uma enfermidade sistêmica idiopática caracterizada por um processo inflamatório não supurativo que acomete a musculatura esquelética e manifesta-se clinicamente por fraqueza muscular proximal e simétrica. Possui incidência variável, cerca de um caso para cada 100 mil habitantes, havendo predominância no sexo feminino. Procedimentos: avaliou-se no Setor de Otoneurologia de uma Instituição, um paciente com diagnóstico de polimiosite, com 60 anos, do sexo feminino, que referiu dificuldade para escutar no ouvido direito, zumbido, ansiedade, insônia, dificuldade nos movimentos do pescoço e dor irradiada para ombros e braços. Foram realizados os seguintes procedimentos: anamnese, inspeção otológica, avaliação audiológica e vestibular. Resultados: a) na avaliação audiológica apresentou, perda auditiva neurossensorial de grau moderado na orelha direita e limiares auditivos dentro dos padrões de normalidade na orelha esquerda; b) na imitanciometria apresentou, curva timpanométrica do tipo " $A$ " e presença dos reflexos estapedianos bilateralmente; c) no exame vestibular observou-se, nistagmo semi-espontâneo do tipo múltiplo e ausência do efeito inibidor da fixação ocular. Conclusão: observou-se alteração nos exames realizados, principalmente, no exame labiríntico que demonstrou a importância de sua realização e a sensibilidade na captação de alteração que sugeriu envolvimento do sistema nervoso central contribuindo dessa forma, para uma intervenção e acompanhamento terapêutico mais rápido e eficaz.
\end{abstract}

DESCRITORES: Polimiosite; Audição; Testes da Função Vestibular; Zumbido; Nistagmo Patológico

(1) Fonoaudióloga; Professora Adjunta do Curso de Fonoaudiologia da Universidade Tuiuti do Paraná, UTP, Curitiba, PR; Coordenadora do Programa de Mestrado e Doutorado em Distúrbios da Comunicação da Universidade Tuiuti do Paraná, UTP, Curitiba, PR; Doutora e Pós-Doutora em Distúrbios da Comunicação Humana pela Universidade Federal de São Paulo.

(2) Fonoaudióloga; Mestre em Distúrbios da Comunicação pela Universidade Tuiuti do Paraná; Doutoranda em Distúrbios da Comunicação da Universidade Tuiuti do Paraná, UTP, Curitiba, PR.

(3) Médico Otorrinolaringologista do Instituto Paranaense de Otorrinolaringologia, IPO, Curitiba, PR; Mestrando em Distúrbios da Comunicação da Universidade Tuiuti do Paraná, UTP, Curitiba, PR.

(4) Médico Neurologista do Departamento de Neurologia Infantil do Hospital Pequeno Príncipe e do Laboratório de EEG Digital do Hospital da Cruz Vermelha Brasileira, HCV, Curitiba, PR; Mestre em Neurociências pela Universidade Federal de São Paulo; Doutorando em Distúrbios da Comunicação da Universidade Tuiuti do Paraná, UTP, Curitiba, PR.

(5) Fonoaudióloga; Mestranda em Distúrbios da Comunicação da Universidade Tuiuti do Paraná, UTP, Curitiba, PR.

Conflito de interesses: inexistente

\section{INTRODUÇÃO}

As miopatias inflamatórias idiopáticas, também conhecidas como "síndrome de miosite", são da família das doenças sistêmicas adquiridas do tecido conjuntivo, caracterizadas por efeitos clínicos e patológicos de inflamação muscular crônica. As formas mais comuns são a polimiosite e a dermatomiosite ${ }^{1-3}$.

A polimiosite (PM), é uma enfermidade sistêmica idiopática, caracterizada por um processo inflamatório não supurativo que acomete o tecido muscular esquelético e manifesta-se clinicamente por fraqueza muscular bilateral e simétrica ${ }^{1-3}$. Diversos estudos ${ }^{1-4}$ referem que ocorre uma resposta anormal imune ao músculo esquelético. Sua fisiopatologia é desconhecida, e, dentre os mecanismos mais prováveis, sugere-se injúria muscular mediada por vírus ou insulto microvascular por auto-antígenos ${ }^{1-4}$. 
Possui incidência variável geograficamente, cerca de um caso para cada 100 mil habitantes, havendo predominância no sexo feminino ${ }^{2,3}$. Sua distribuição mundial chega a atingir um pico de prevalência na primeira década de vida e outro entre os 50-60 anos de idade ${ }^{1,3}$.

Os indivíduos de descendência africana e hispânica estão sujeitos a ter um risco aumentado de miopatias inflamatórias idiopáticas com prognóstico mais grave quando comparados com indivíduos da raça branca ${ }^{2}$.

O início é frequentemente gradual e progressivo, com meses ou anos de evolução. O comprometimento da musculatura esquelética da parede posterior da faringe e do terço proximal do esôfago podem dar origem à disfonia e a disfagia com refluxo de alimentos para vias aéreas superiores devido à fraqueza muscular cricofaríngea. O comprometimento do flexor do pescoço ocorre em dois terços dos casos, o que pode ocasionar dificuldade para sustentar o pescoço. Além da fraqueza muscular que é o seu sintoma principal, acomete músculos da cintura escapular e pélvica, progredindo para a musculatura proximal dos membros. Outros sintomas observados incluem: fadiga, astenia, mialgias, febre, perda de peso, artralgia e artrite de pequenas e médias articulações. O envolvimento cardíaco pode estar presente em até $30 \%$ dos casos e geralmente se traduz por taquicardia, arritmias, defeitos da condução átrio-ventricular e bloqueios de ramos. Raramente há insuficiência cardíaca congestiva, seja por miocardite ou por fibrose miocárdica $2,3,5,6$. As manifestações pulmonares são provavelmente as manifestações sistêmicas da doença mais comuns e sua prevalência é relatada em 5 a $46 \%$ dos casos ${ }^{6}$ sendo, as mais comuns, a pneumonia aspirativa, hipoventilação por fraqueza muscular, infecções e doença intersticial. Esta última afeta $5 \mathrm{a}$ $10 \%$ dos pacientes.

O diagnóstico entre as miopatias inflamatórias baseia-se no quadro clínico da fraqueza muscular, exames laboratorias com alteração nos níveis de enzimas musculares, exame eletroneuromiográfico, biópsia muscular e na resposta ao tratamento ${ }^{2,3,5,6}$.

Com relação ao sistema auditivo, o mesmo pode não apresentar alteração ou, estar vulnerável às alterações patológicas tanto local quanto sistêmica. As alterações estruturais e bioquímicas podem atuar independentemente ou associadas, comprometendo os mecanismos de audição e equilíbrio ${ }^{2}$.

O equilíbrio corporal depende da integridade do sistema vestibular (labirinto, nervo vestibulococlear, núcleos, vias e inter-relações no sistema nervoso central - SNC), do sistema somatossensorial (receptores sensoriais localizados em tendões, músculos e articulações) e da visão sob a coordenação do cerebelo. Se houver uma lesão em algum destes sistemas, ocorrerá um conflito de informações, podendo surgir sintomas de desequilíbrio corporal e tontura ${ }^{7}$.

O sistema vestibular periférico é constituído pelas células ciliadas das cristas ampulares dos ductos semicirculares, células ciliadas das máculas utriculares e saculares do labirinto membranáceo, fibras nervosas aferentes e eferentes, gânglio de Scarpa, nervos vestibulares superior e inferior e anastomoses vestibulofaciais e vestibulococleares $^{8}$.

O sistema vestibular central é composto pelos núcleos vestibulares no soalho do IV ventrículo do tronco encefálico, vias vestibulooculomotoras, vestibulocerebelares, vestibulocortical, vetibuloespinal, vestibulovagal, vestibulares eferentes e inter-relações com a formação reticular e outras áreas do SNC ${ }^{8}$.

Sinais dos receptores proprioceptivos cervicais, localizados nos ligamentos e juntas das vértebras cervicais superiores, interagem nos núcleos vestibulares com sinais vestibulares aferentes. A estimulação unilateral destes receptores proprioceptivos produz ativação nuclear vestibular contralateral. A interrupção unilateral dos sinais proprioceptivos cervicais por secção radicular ou bloqueio pode originar nistagmo e vertigem ${ }^{5}$. Ou seja, o comprometimento em diversas áreas, interligadas com estruturas do sistema vestibular, principalmente as vias vestibuloespinais, podem causar alterações labirínticas com envolvimento do SNC.

A avaliação otoneurológica é um conjunto de procedimentos que permite a exploração semiológica dos sistemas auditivo e vestibular e de suas relações com o sistema nervoso central ${ }^{9}$.

Diante do exposto, o objetivo do presente estudo foi apresentar os achados vestibulococleares em um caso com diagnóstico de PM e ressaltar a importância e a sensibilidade do exame vestibular em captar alterações que sugiram envolvimento do SNC.

\section{APRESENTAÇÃO DO CASO}

Avaliou-se uma paciente do sexo feminino com diagnóstico de PM de 60 anos de idade, encaminhada ao Setor de Otoneurologia de uma Instituição na cidade de Curitiba/Pr.

Realizaram-se os seguintes procedimentos:

\section{Anamnese}

Aplicou-se um questionário com ênfase aos sinais e sintomas audiológicos, antecedentes pessoais e familiares. 


\section{Avaliação Otorrinolaringológica}

Realizada pelo médico da clínica de fonoaudiologia com o objetivo de excluir qualquer alteração que pudesse interferir no exame.

\section{Avaliação Audiológica}

A audiometria tonal limiar convencional foi realizada com um audiômetro de 2 canais, da marca Madsen-GN Otometrics, modelo Itera, com fones TDH-39, com limiares em dB NA. O equipamento encontra-se calibrado de acordo com o padrão ISO 8253. A seguir, pesquisou-se a determinação do limiar de reconhecimento de fala (LRF) e do índice percentual de reconhecimento de fala (IPRF), em cabine acusticamente tratada para impedir a interferência de ruídos estranhos ao teste. Foram aplicados critérios para caracterização do grau e tipo de perda auditiva ${ }^{10}$.

\section{Medidas de Imitância Acústica}

Este procedimento foi realizado para avaliar a integridade do sistema tímpano-ossicular por meio da curva timpanométrica e da pesquisa do reflexo acústico. $O$ equipamento utilizado foi o impedanciômetro Madsen Otoflex 100. Aplicou-se critérios para a interpretação dos resultados ${ }^{11}$.

\section{Avaliação Vestibular}

O paciente foi submetido às seguintes provas que compõem o exame vestibular:

Pesquisou-se a vertigem e os nistagmos de posição/posicionamento, espontâneo e semi-espontâneo.

\section{Com registro}

Para a realização da vectoeletronistagmografia utilizou-se um aparelho termossensível, com três canais de registro, da marca Berger, modelo VN316. Colocaram-se, fixados com pasta eletrolítica, um eletródio ativo no ângulo lateral de cada olho e na linha média frontal, formando um triângulo isósceles, que permite a identificação dos movimentos oculares horizontais, verticais e oblíquos e principalmente para o cálculo da velocidade angular da componente lenta do nistagmo (VACL).

Utilizou-se uma cadeira rotatória pendular decrescente da marca Ferrante, um estimulador visual modelo EV VEC e um otocalorímetro a ar modelo NGR 05, ambos da marca Neurograff.

Realizaram-se as seguintes provas oculares e labirínticas à VENG, de acordo com critérios anteriormente propostos ${ }^{12}$.

"Calibração dos movimentos oculares, pesquisa dos nistagmos espontâneo e semi-espontâneo, pesquisa do rastreio pendular, pesquisa dos nistagmos optocinético, per-rotatório pelos canais posteriores direito e esquerdo e pré e pós-calórico. O tempo de estimulação calórica em cada orelha com ar a $42^{\circ} \mathrm{C}$ e $18^{\circ} \mathrm{C}$ durou 80 s para cada temperatura e as respostas foram registradas com os olhos fechados e, a seguir, com os olhos abertos para a observação do efeito inibidos da fixação ocular.

A pesquisa foi realizada após autorização do paciente por meio de assinatura do Termo de Consentimento Livre e Esclarecido.

\section{RESULTADOS}

As queixas referidas na anamnese foram: dificuldade para escutar no ouvido direito, zumbido, ansiedade, insônia, dificuldade nos movimentos do pescoço, dor irradiada para ombros e/ou braços.

O diagnóstico da PM foi realizado no início do ano 2000 , sendo medicada e acompanhada desde essa época.

Avaliação Audiológica - apresentou perda auditiva do tipo neurossensorial de grau moderado na orelha direita (OD) e limiares auditivos dentro dos padrões de normalidade na orelha esquerda (OE).

Medidas de Imitância Acústica - apresentou curvas timpanométricas do tipo " $A$ " com presença de reflexo acústico bilateralmente.

\section{Avaliação vestibular}

- Nistagmo e vertigem de posição e/ou posicionamento: ausente;

- Calibração dos movimentos oculares: regular;

- Nistagmo espontâneo: olhos abertos: ausente; olhos fechados: presente, do tipo horizontal para esquerda com VACL $2 \%$;

- Nistagmo semi-espontâneo: presente do tipo múltiplo - horizontal para a esquerda, não fatigável, sem paroxismo, sem latência, com VACL 7\%/s;

- Rastreio pendular: Tipo II;

- Nistagmo optocinético: simétrico com VACL $\mathrm{AH}=7 \% \mathrm{~s} ; \mathrm{VACL} \mathrm{H}=7 \%$;

- Nistagmo per-rotatório: estimulação dos ductos semicirculares laterais: simétricos com freqüência nistágmica: $\mathrm{AH}=7 ; \mathrm{H}=9 ; \mathrm{PDN}=12 \%$ à direita. Estimulação dos ductos semicirculares posteriores: simétricos com freqüência nistágmica: $\mathrm{AH}=9 ; \mathrm{H}=10 ; \mathrm{PDN}=5 \%$ à direita. Estimulação dos ductos semicirculares anteriores: simétricos com freqüência nistágmica: $\mathrm{AH}=10$; $\mathrm{H}=3 ; \mathrm{PDN}=54 \%$ à esquerda.

- Nistagmo pós-calórico: prova calórica realizada comar; $42^{\circ} \mathrm{COD}: \mathrm{VACL}=3 \%$ /s; $42^{\circ} \mathrm{COE}: \mathrm{VACL}=5 \%$; $18^{\circ} \mathrm{C}$ OD: $\mathrm{VACL}=7 \% \mathrm{~s} ; 18^{\circ} \mathrm{C}$ OE: $\mathrm{VACL}=9 \%$

- Predomínio Labiríntico (PL) $17 \%$ à esquerda com presença de EIFO nas quatro estimulações. 
- Achados no exame: nistagmo semi-espontâneo do tipo múltiplo com características centrais e ausência de EIFO.

- Conclusão do exame: Sugestivo de Síndrome Central.

\section{DISCUSSÃO}

O equilíbrio corporal depende da integridade do sistema vestibular: labirinto, nervo vestibulococlear, núcleos, vias e inter-relações no sistema nervoso central, do sistema somatossensorial e da visão. Tonturas e/ou desequilíbrio surgem quando algo interfere no funcionamento normal do sistema de equilíbrio corporal ${ }^{6}$. O estudo do nistagmo realizado por meio da VENG tem-se constituído como um recurso preciso no topodiagnóstico de várias doenças ${ }^{12}$.

No caso relatado, observam-se alguns sinais centrais como: presença de nistagmo do tipo múltiplo na pesquisa do nistagmo semi-espontâneo, sendo este visível e registrável com características centrais, ou seja, sem latência, paroxismo, fatigabilidade e vertigem e, ausência de EIFO nessa prova em comparação com os olhos fechados.

$\mathrm{Na}$ literatura compulsada com relação à parte otoneurológica não foram encontrados estudos relacionados à $\mathrm{PM}$. Observaram-se dois relatos de caso dos autores ${ }^{1}$ que referiram em uma paciente do sexo feminino de 66 anos de idade, fortes dores na região abdominal e nos membros superiores e inferiores além de referir diminuição de força muscular dos mesmos, dispneia que piorava aos esforços associada com tosse e febre.
Num segundo caso, os autores relataram, outra paciente do sexo feminino de 54 anos de idade, com diagnóstico de hipotiroidismo, que relatou aparecimento de lesão com característica linear, esbranquiçada na língua, inicialmente pequena e que progressivamente passou a ocupar toda a língua e mucosa oral. Associado ao quadro, a paciente referiu glossalgia, disfagia, perda do paladar e parestesia em cavidade oral e região perioral. Houve perda ponderal de $15 \mathrm{~kg}$ nos últimos seis meses, astenia desde o início do quadro que progressivamente evoluiu para sensação de fraqueza muscular nos membros superiores e inferiores e dor na região do pescoço.

Em outro caso, os autores ${ }^{13}$ relatam paciente também do sexo feminino, de 19 anos de idade que foi internada com quadro de mialgia, astenia, náusea, vômito, apresentava força muscular grau II e dores musculares difusas de evolução há três meses. A incapacidade de deglutição obrigou a instalação de sonda nasogástrica. Havia relato de picos febris e uso de amoxicilina sem resolução dos sintomas.

\section{CONCLUSÃO}

Observou-se alteração nos exames realizados, principalmente, no exame labiríntico que demonstrou a importância de sua realização e a sensibilidade na captação de alteração que sugeriu envolvimento do sistema nervoso central contribuindo dessa forma, para uma intervenção e acompanhamento terapêutico mais rápido e eficaz. 


\begin{abstract}
Background: polymyositis is an idiopathic systematic disease characterized by a non-suppurative inflammatory process which attacks the skeletal musculature and manifests itself clinically by proximal and symmetrical muscular debility. It shows a variable incidence, approximately one in every hundred thousand inhabitants, predominately in females. Procedures: a patient, 60 years, female and diagnosed with polymyositis was evaluated in the Otoneurology Sector of an Institution, referred having hearing difficulties in the right ear, tinnitus, anxiety, insomnia, difficulties in neck movement and shoulders and arms disseminated pain. The following procedures were carried out: anamnesis, otological inspection, audiological evaluation and vestibular exam. Results: a) in the audiological evaluation there was sensorineural hearing loss from moderate degrees in the right ear and normal hearing thresholds in the left ear; $b$ ) in the imitanciometry there was a type " $A$ " tympanometric curve and the presence of the stapedial reflexes, bilaterally; $c$ ) in the vestibular exam, semi-spontaneous multiple nystagmus and an absence of the inhibiting effect for ocular fixation were observed. Conclusion: alterations were observed in the accomplished exams, mainly in the vestibular exam, which demonstrated the importance for being carried out; and the sensitivity in the acquisition of the alteration which suggested an involvement of the central nervous system, contributing, thus, to a faster and more effective intervention and therapeutic accompaniment.
\end{abstract}

KEYWORDS: Polymyositis; Hearing; Vestibular Function Tests; Tinnitus; Nistagmus, Pathologic

\section{REFERÊNCIAS}

1. Costa LFC, Tavares FFP, Nunes MLA. Miopatias inflamatórias idiopáticas: relato de caso. Rev Bras Clin Med. 2009; 7:53-5.

2. Rachid A, Magalhães FLGM, Parchen CFR, Amaral VCQ, Lopata O. Um caso atípico de polimiosite com acometimento da língua. Rev Bras Reumatol. 2005; 45(4):270-2.

3. Reis Neto ET, Pollak DF. Miopatias inflamatórias no idoso. Eisntein. 2008; 6(Supl1):548-54.

4. Karnikowski MGO, Costa BRV, Osella OFS, Nóbrega OT. Polymyositis: clinical investigation in two sisters. Arq Neuropsiquiatr. 2002; 60(3-A):624-7.

5. Hengstman GL, van Engelen BG. Polymyositis, invasion of non-necrotic muscle fibres, and the art of repetition. BMJ. 2004; 329(7480):1464-7.

6. Fathi M, Dastmalchi M, Rasmussen E, Lundberg

E, Tomling G. Interstitial lung disease, common manifestation of newly diagnosed polymyosistis and dermatomyositis. Ann Rheum Dis. 2004; 63(3):297-301.

RECEBIDO EM: 14/10/2009

ACEITO EM: 08/03/2010

Endereço para Correspondência:

Bianca Simone Zeigelboim

Rua Gutemberg, 99 9 and

Curitiba - PR

CEP: 80420-030

E-mail: bianca.zeigelboim @utp.br
7. Ganança MM, Munhoz MSL, Caovilla HH, Silva MLG, Ganança FF, Perracini MR, et al. Conceitos e algoritmos terapêuticos. In: Ganança MM, Munhoz MSL, Caovilla HH. Silva MLG. Condutas na vertigem. São Paulo: Grupo Editorial Moreira; 2004.p. 60-75. ou 55-112

8. Ganança MM, Caovilla HH, Munhoz MSL, Garcia da Silva ML, Ganança FF, Ganança CF. A hodologia clínica do sistema vestibular. In: Caovilla HH, Ganança MM, Munhoz MSL, Garcia da Silva ML. Equilibriometria clínica. São Paulo: Atheneu; 2000. p.5-21.

9. Ganança MM, Caovilla HH, Munhoz MSL, Silva MLG, Ganança FF, Ganança CF. A vertigem explicada. RBM Especiais [periódico na Internet] Nov 1999 [acesso em set 2009]; 56: [27]. Disponível em: http://www.cibersaude.com.br/revistas. asp? fase $=$ r003\&id_materia $=421$.

10. Davis $\mathrm{H}$, Silverman RS. Hearing and deafness. 3. ed. New York: Holt, Rinehart \& Wilson; 1970. p.253-79.

11. Jerger J. Clinical experience with impedance audiometry. Arch Otolaringol 1970; 92:311-24.

12. Mangabeira-Albernaz PL, Ganança MM, Pontes PAL. Modelo operacional do aparelho vestibular. In: Mangabeira-Albernaz PL, Ganança MM. Vertigem. 2.ed. São Paulo: Moderna; 1976. p. 29-36.

13. Muller CS, Rachid Filho A, Titton DC, Paiva ES. Manifestações clínicas e laboratoriais de polimiosite em um caso de leptospirose. Rev Bras Reumatol. 2006; 46(6):424-7. 